\title{
STUDY AND ANALYSIS OF EFFECT OF STRENGTH PROPERTIES IN CALCINED KAOLIN AND SILICA FUME
}

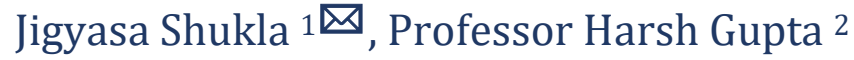 \\ ${ }^{1}$ Research Scholar, Department of Civil Engineering, Jawaharlal Nehru College of Technology, \\ Rewa (M.P.), India \\ 2 Professor, Department of Civil Engineering, Jawaharlal Nehru College of Technology, Rewa \\ (M.P.), India
}

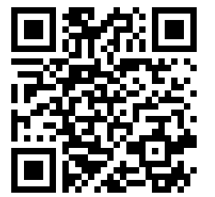

DOI: https://doi.org/10.29121/granthaalayah.v8.i6.2020.575

Article Type: Case Study

Article Citation: Jigyasa Shukla, and Professor Harsh Gupta. (2020). STUDY AND ANALYSIS OF EFFECT OF STRENGTH PROPERTIES IN CALCINED KAOLIN AND SILICA FUME. International Journal of Research -GRANTHAALAYAH, 8(6), 263-269. https://doi.org/10.29121/granthaa layah.v8.i6.2020.575

Received Date: 11 May 2020

Accepted Date: 30 June 2020

Keywords:

Silica Fume

Durability

Compressive Strength

Flexural Strength

\begin{abstract}
This paper present the study of various strength such as compressive strength, split tensile strength and flexural strength during 7 and 28 day. It is construct the specimens size $15 \mathrm{~cm} X 15 \mathrm{~cm}$ X $15 \mathrm{~cm}$ for testing purpose which depend upon the size of aggregate. Test results are indicated that strength performance of concrete well as in durability aspect are improved using of Silica fume.
\end{abstract}

\section{INTRODUCTION}

Silica fume, durability and Compressive strength etc are studying and analyze. The mix (M1, M2, M3, M4 and M5) are used to analyze and absorb the optimum mix for construction using of some Calcined Kaolin, Sand and Aggregate.

\section{COMPRESSIVE STRENGTH FORMULA}

Compressive Strength $=$ Load $/$ Cross-sectional Area 


\section{PROCEDURE}

The cubical moulds of size $15 \mathrm{~cm} \times 15 \mathrm{~cm} \times 15 \mathrm{~cm}$ are used for testing and analyzing. The concrete is poured and mould with tempered properly to remove any voids, after this 24-hour cubical moulds are removed and test specimens are putting in water for curing purpose. All specimens are tested with the help of compression testing machine after 7 days curing and 28 days curing. The load $\left(150 \mathrm{~kg} / \mathrm{cm}^{2}\right.$ per minute) are applied gradually at till the Specimens fails.

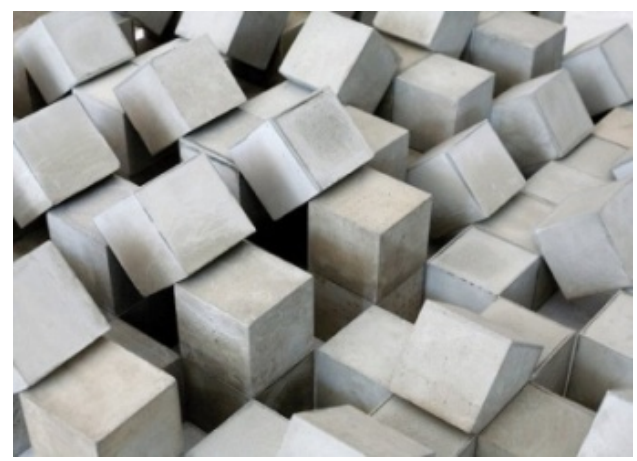

Figure 1: Specimens

\section{PREPARATION}

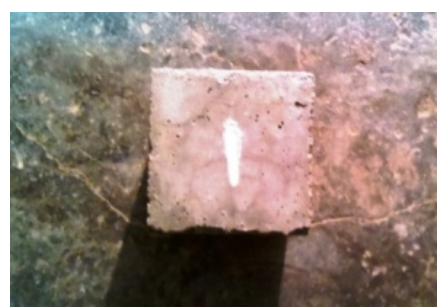

Figure 2: First Specimen

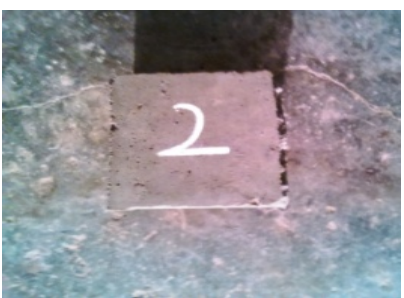

Figure 3: Second Specimen

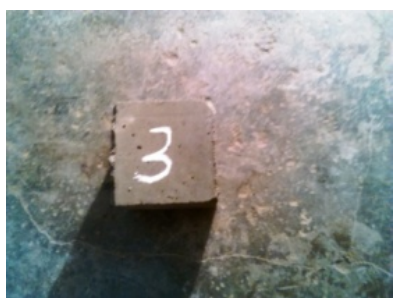

Figure 4: Third Specimen

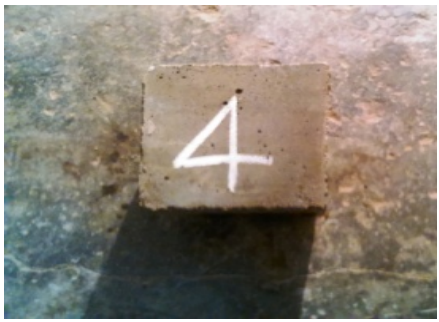

Figure 5: Fourth Specimen

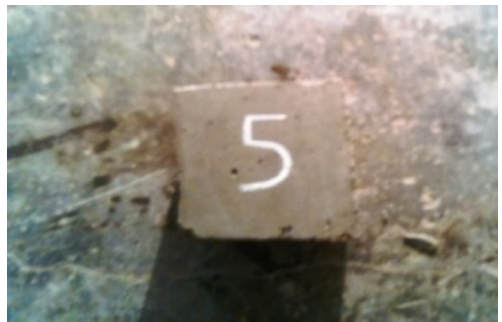

Figure 6: Fifth Specimen

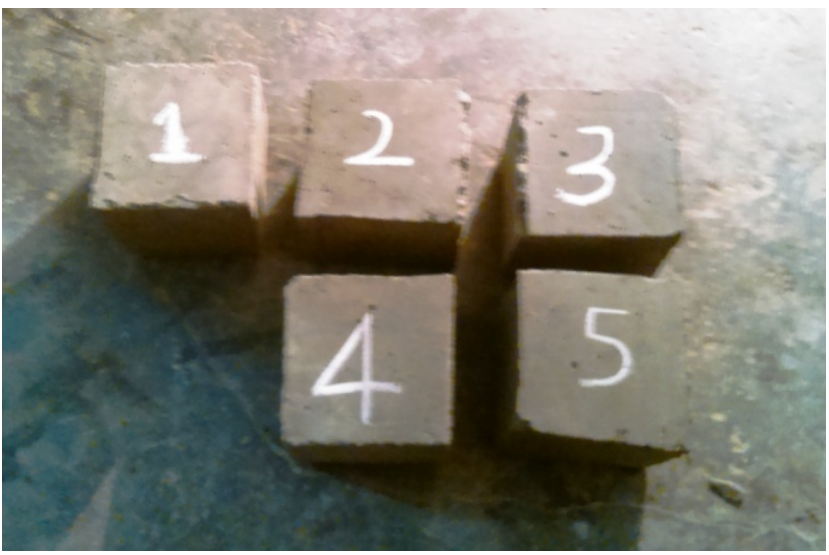

Figure 7: First to Fifth Specimens 


\section{RESULTS AND DISCUSSION}

Table 1: Properties of Cement

\begin{tabular}{|c|c|c|}
\hline Sr. No. & Property & Results \\
\hline 1 & Normal Consistency & $34 \%$ \\
\hline 2 & Initial Setting time & 50 minutes \\
\hline 3 & Specific Gravity & 3.15 \\
\hline 4 & Fineness of cement & $5 \%$ \\
\hline
\end{tabular}

Table 2: Properties of Fine Aggregate

\begin{tabular}{|c|c|c|}
\hline Sr. No. & Property & Results \\
\hline 1 & Specific Gravity & 2.57 \\
\hline 2 & Fineness modulus & 2.28 \\
\hline
\end{tabular}

Table 3: Properties of Silica Fume

\begin{tabular}{|c|c|c|}
\hline Sr. No. & Property & value \\
\hline 1 & Specific Gravity & 2.2 \\
\hline 2 & Bulk Density & $576,\left(\mathrm{Kg} / \mathrm{m}^{3}\right)$ \\
\hline 3 & Size, (Micron) & 0.1 \\
\hline 4 & Surface Area, $\left(\mathrm{m}^{2} / \mathrm{kg}\right)$ & 20,000 \\
\hline 5 & $\mathrm{Si02}$ & $90 \%-96 \%$ \\
\hline 6 & $\mathrm{Al} 203$ & $0.5 \%-0.8 \%$ \\
\hline
\end{tabular}

Table 4: Properties of Super Plasticizer

\begin{tabular}{|c|c|c|}
\hline Sr. No. & Property & value \\
\hline 1 & Specific Gravity & $1.220-1.225$ \\
\hline 2 & Chloride content & NIL \\
\hline 3 & Air entrainment & approximately $1 \%$ additional air \\
\hline
\end{tabular}

Table 5: Mix Proportioning

\begin{tabular}{|c|c|c|}
\hline Sr. No. & Material & Quantity in $\mathrm{Kg} / \mathrm{m}^{3}$ \\
\hline 1 & Cement (OPC) & 514 \\
\hline 2 & Fine Aggregate & 456.932 \\
\hline 3 & Coarse Aggregate & 1391.642 \\
\hline 4 & Water & 185.6 \\
\hline
\end{tabular}

Table 6: Results of Compressive Strength (7 days)

\begin{tabular}{|c|c|c|}
\hline Mix & \% of Silica Silica Fume added \% & Compressive Strength $\left(\mathrm{N} / \mathrm{mm}^{2}\right)$ \\
\hline M1 & 0 & 26.21 \\
\hline M2 & 5 & 30.22 \\
\hline M3 & 10 & 36.45 \\
\hline M4 & 15 & 39.24 \\
\hline M5 & 20 & 38.55 \\
\hline
\end{tabular}


Study and Analysis of Effect of Strength Properties in Calcined Kaolin and Silica Fume

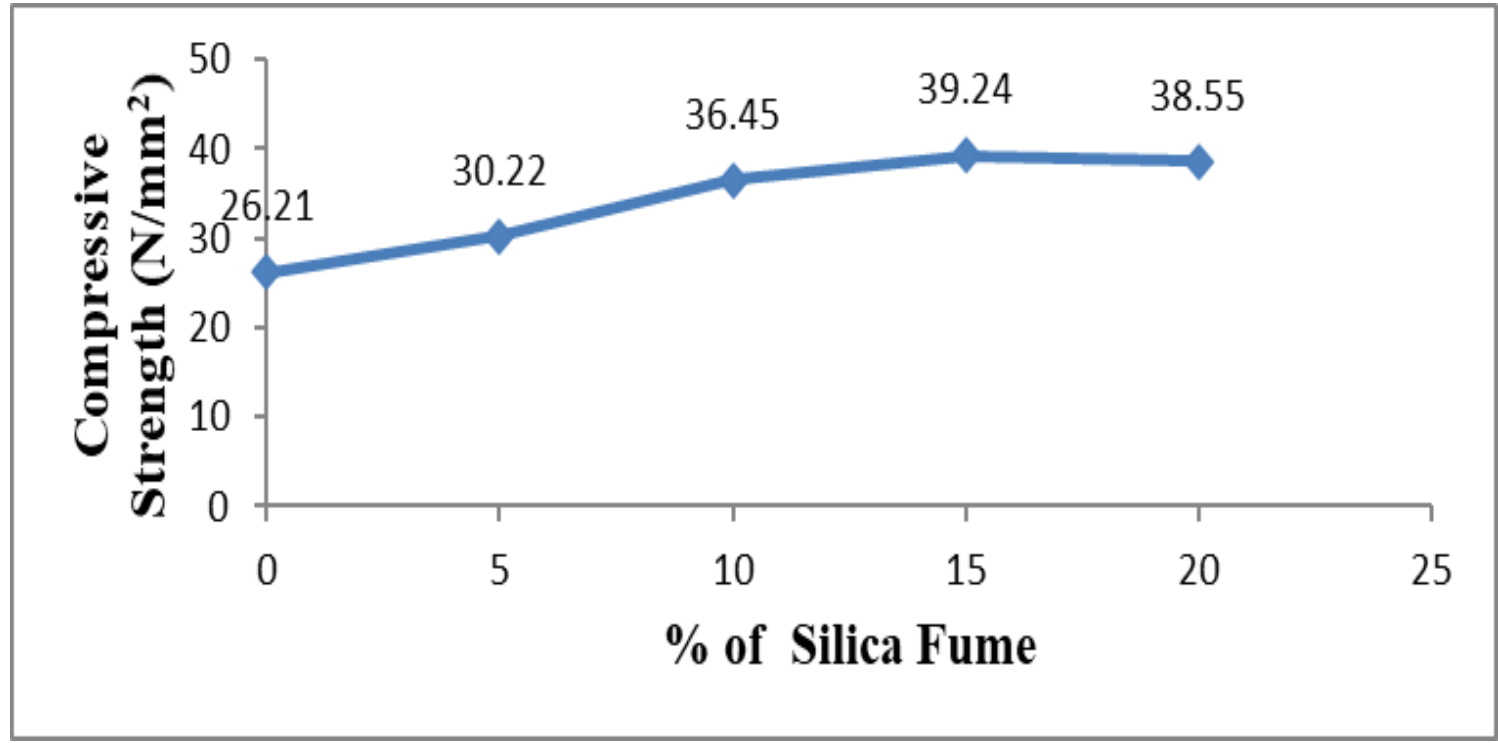

Figure 8: Results of Compressive Strength (7 days)

Table 7: Results of Compressive Strength (28 days)

\begin{tabular}{|c|c|c|}
\hline Mix & \% of Silica Silica Fume added \% & Compressive Strength $\left(\mathrm{N} / \mathrm{mm}^{2}\right)$ \\
\hline M1 & 0 & 39.30 \\
\hline M2 & 5 & 42.21 \\
\hline M3 & 10 & 47.89 \\
\hline M4 & 15 & 48.33 \\
\hline M5 & 20 & 45.69 \\
\hline
\end{tabular}

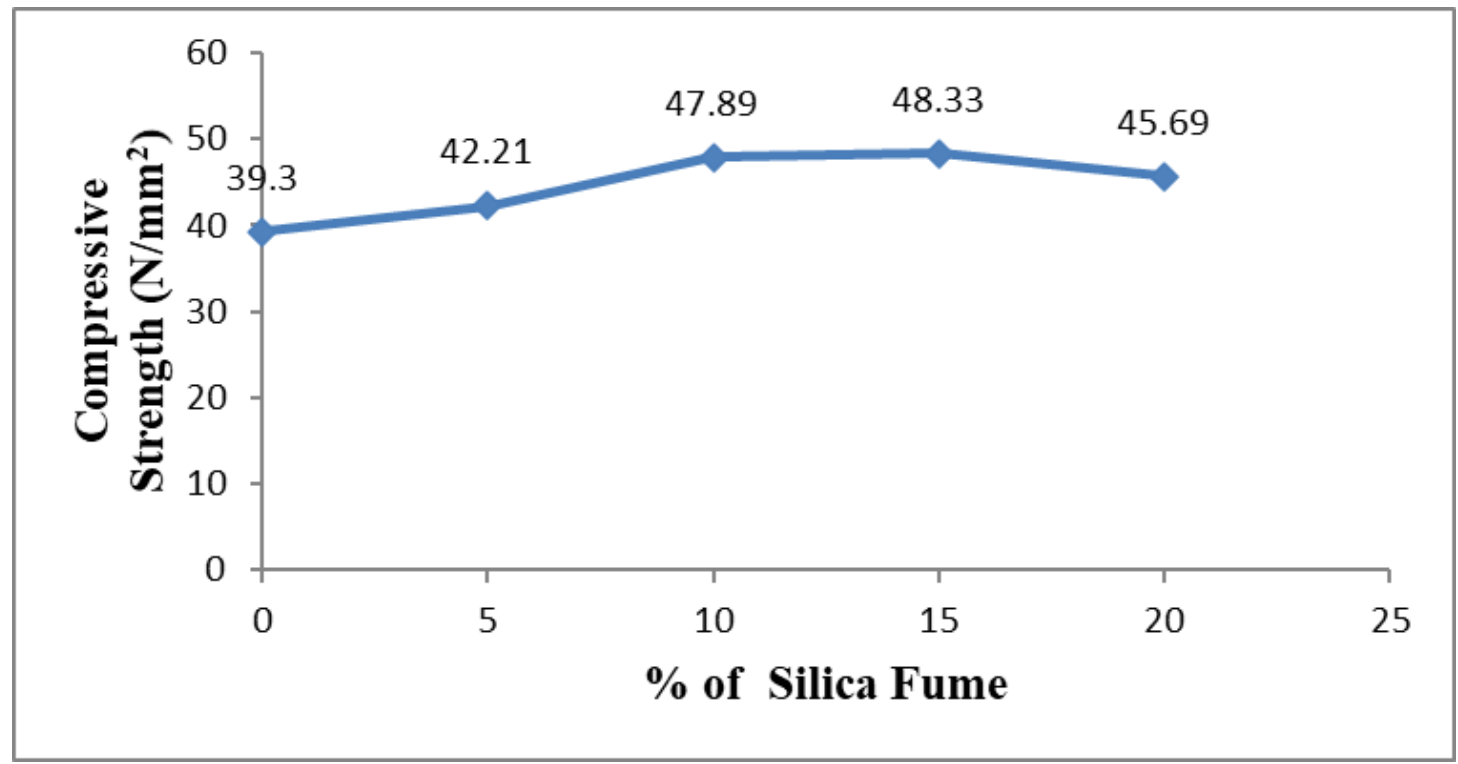

Figure 9: Results of Compressive Strength (28 days)

Table 8: Results of Split tensile Strength (7 days)

\begin{tabular}{|c|c|c|}
\hline Mix & \% of Silica Silica Fume added \% & Split tensile Strength $\left(\mathrm{N} / \mathrm{mm}^{2}\right)$ \\
\hline M1 & 0 & 3.22 \\
\hline M2 & 5 & 3.45 \\
\hline M3 & 10 & 4.65 \\
\hline M4 & 15 & 4.88 \\
\hline M5 & 20 & 3.69 \\
\hline
\end{tabular}


Jigyasa Shukla, and Professor Harsh Gupta

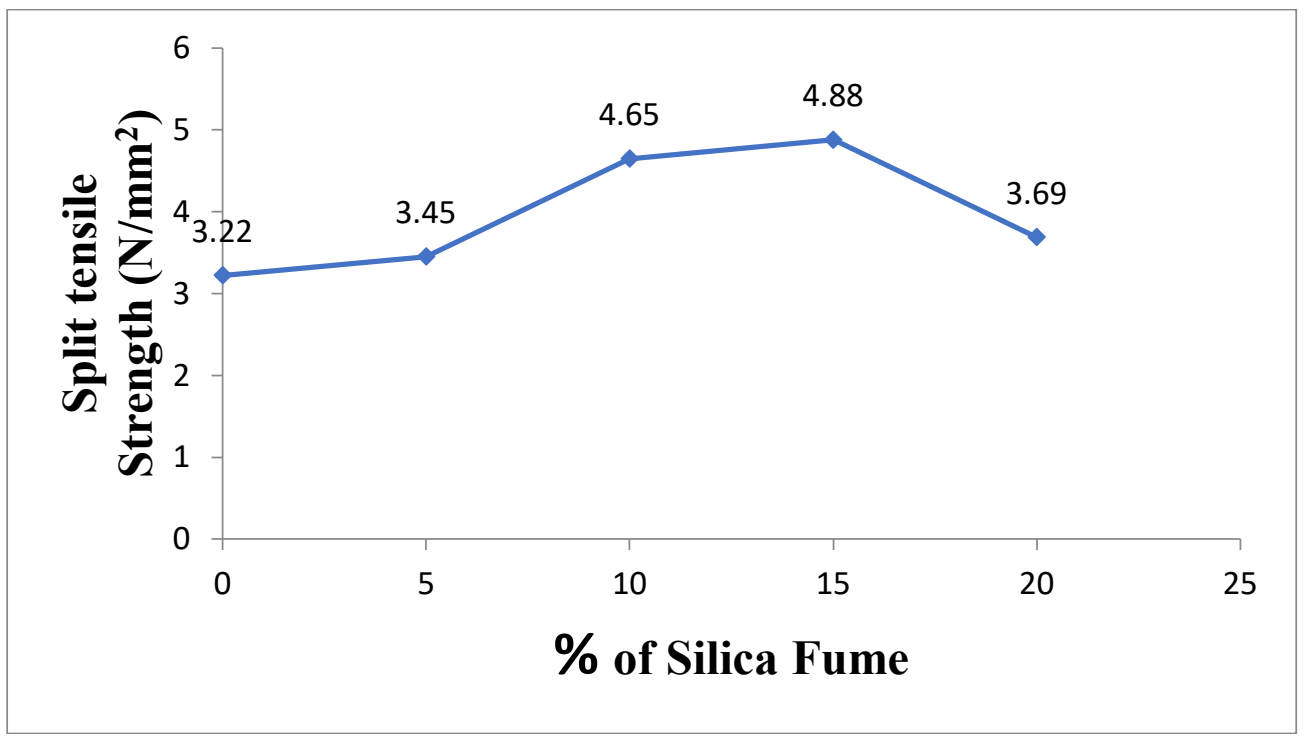

Figure 10: Results of Split tensile Strength (7 days)

Table 9: Results of Split tensile Strength (28 days)

\begin{tabular}{|c|c|c|}
\hline Mix & \% of Silica Silica Fume added \% & Split tensile Strength $\left(\mathrm{N} / \mathrm{mm}^{2}\right)$ \\
\hline M1 & 0 & 4.67 \\
\hline M2 & 5 & 4.71 \\
\hline M3 & 10 & 4.88 \\
\hline M4 & 15 & 4.98 \\
\hline M5 & 20 & 4.22 \\
\hline
\end{tabular}

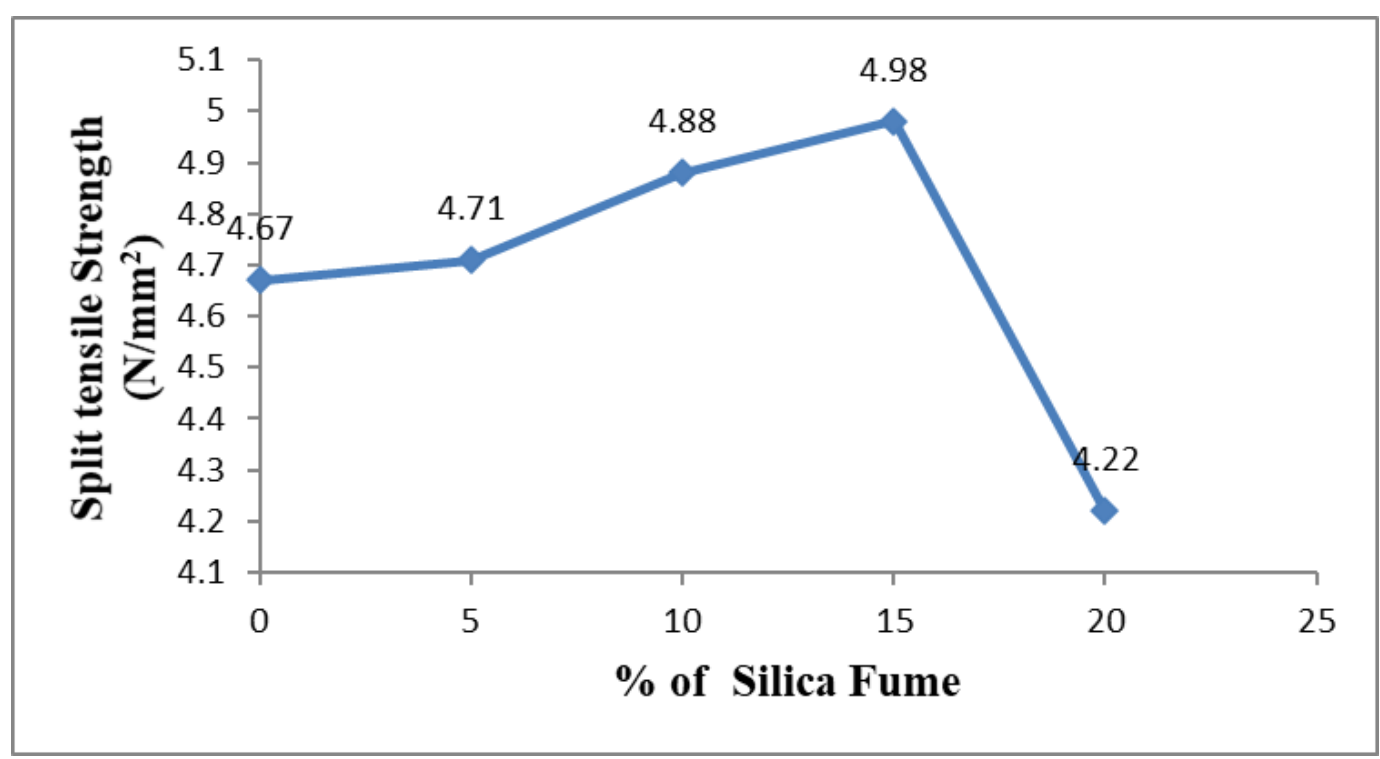

Figure 11: Results of Split tensile Strength (28 days)

Table 10: Results of Flexural Strength (7 days)

\begin{tabular}{|c|c|c|}
\hline Mix & \% of Silica Silica Fume added \% & Flexural Strength $\left(\mathrm{N} / \mathrm{mm}^{2}\right)$ \\
\hline M1 & 0 & 4.33 \\
\hline M2 & 5 & 7.55 \\
\hline M3 & 10 & 7.45 \\
\hline M4 & 15 & 7.89 \\
\hline M5 & 20 & 6.22 \\
\hline
\end{tabular}


Study and Analysis of Effect of Strength Properties in Calcined Kaolin and Silica Fume

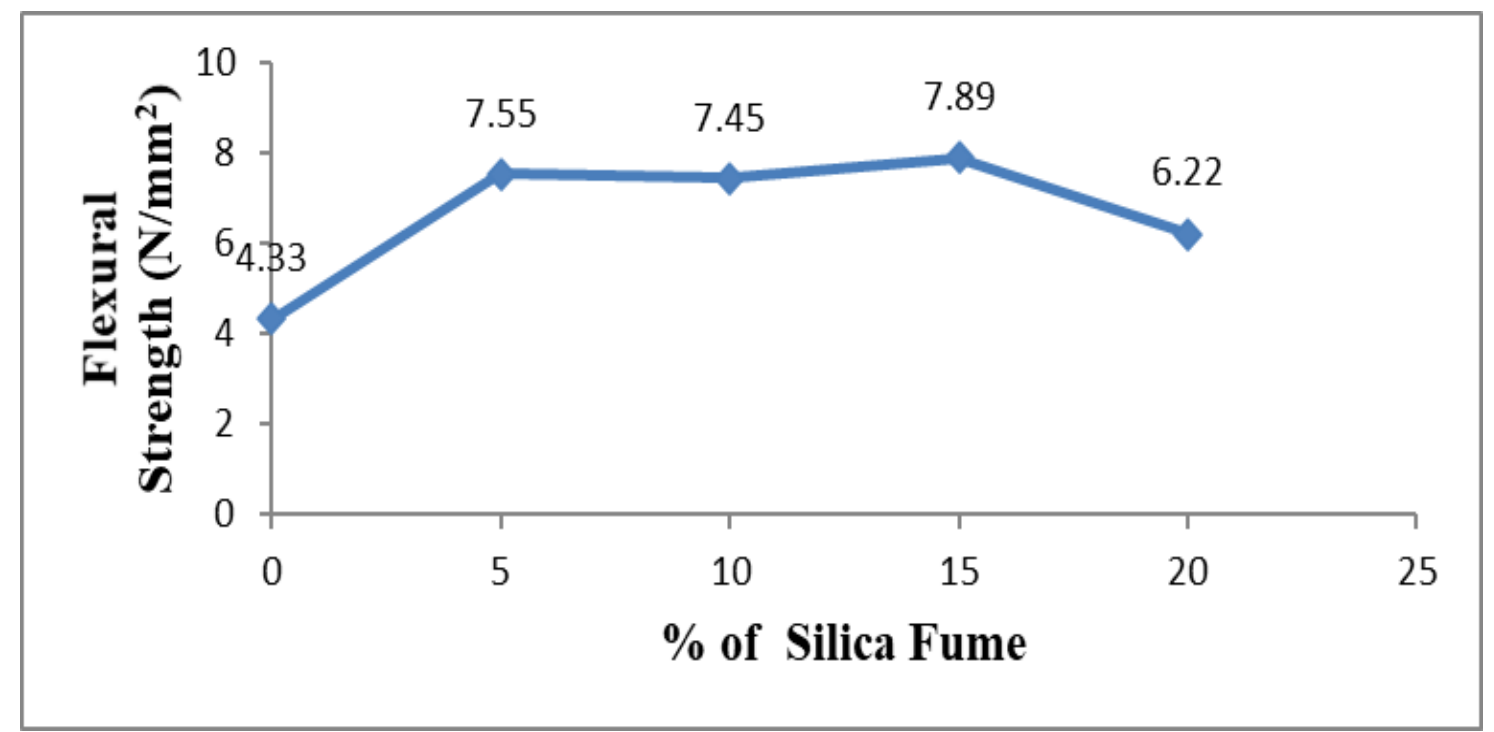

Figure 12: Results of Flexural Strength (7 days)

Table 11: Results of Flexural Strength (28 days)

\begin{tabular}{|c|c|c|}
\hline Mix & \% of Silica Silica Fume added \% & Flexural Strength(N/mm² \\
\hline M1 & 0 & 6.32 \\
\hline M2 & 5 & 7.85 \\
\hline M3 & 10 & 9.88 \\
\hline M4 & 15 & 9.98 \\
\hline M5 & 20 & 7.84 \\
\hline
\end{tabular}

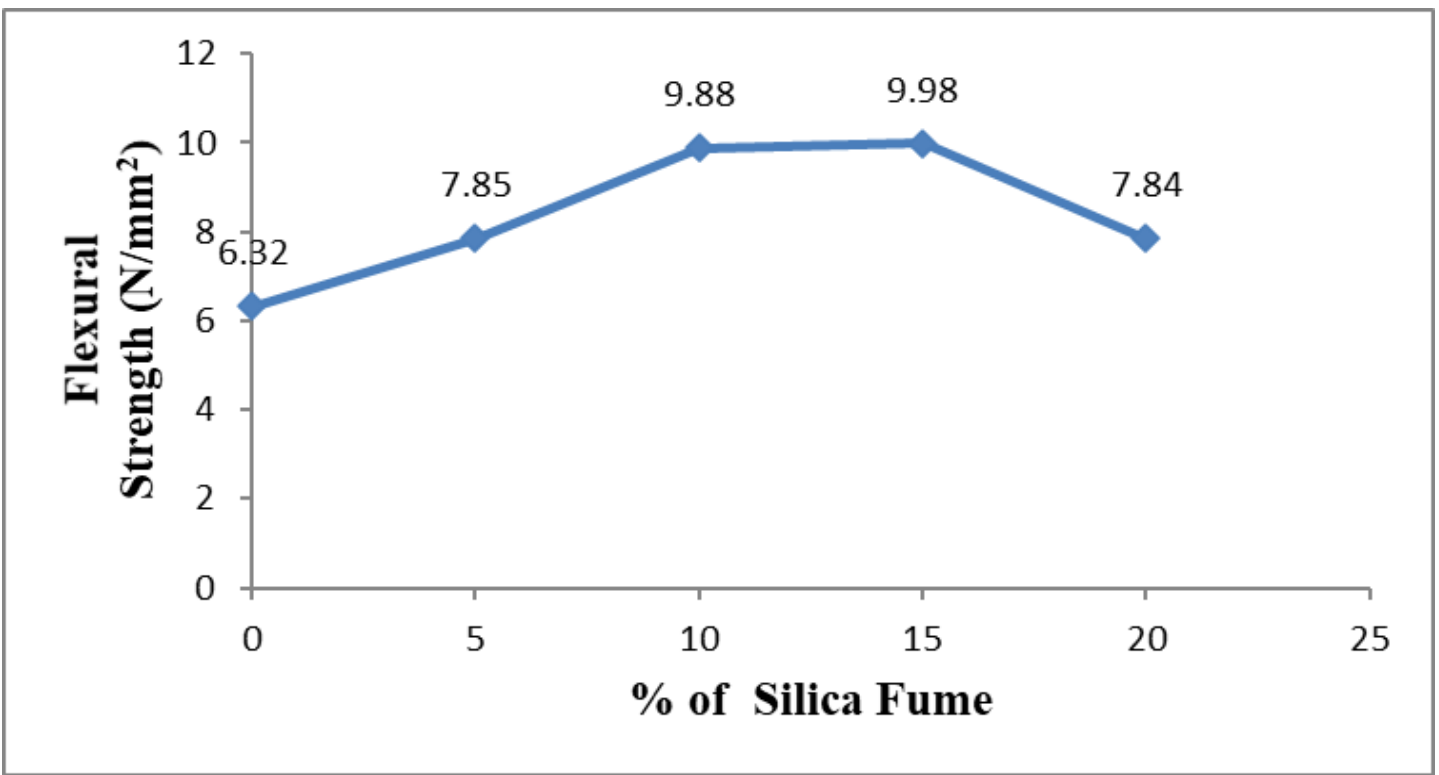

Figure 13: Results of Flexural Strength (28 days)

\section{CONCLUSIONS}

We are construct the specimens size $15 \mathrm{~cm}$ X $15 \mathrm{~cm}$ X $15 \mathrm{~cm}$ which depend upon the size of aggregate. These specimens is poured in the mould and tempered properly during 24 hours. These specimens are tested using of compression testing machine after 7 days curing and after 28 days curing, after this optimum result are finding out using of Mix (M4) which is shown Table 6-11, and Figure 8-13. 


\section{SOURCES OF FUNDING}

None.

\section{CONFLICT OF INTEREST}

None.

\section{ACKNOWLEDGMENT}

None.

\section{REFERENCES}

[1] K. H. 1995. "Effects of anti-washout admixtures on fresh concrete properties." ACI Mater. J., pp 164-171.

[2] Assaad, J. 2003. "Relationship between washout resistance and rheological properties of high-performance underwater concrete." ACI Mater. J., pp 185-193.

[3] Ballivy, G. 1996. "High-performance cement grout for underwater crack injection." Proc., 3rd CANMET/ACI Int. Conf. on Performance of Concrete in Marine Environment, V. M. Malhotra, ed., ACI, Farmington Hills, Mich., pp 138-162.

[4] Hadriche, M. S. 1999. "Factorial design models for proportioning self-consolidating concrete." Mater. Struct., pp 679-686. 